\title{
EXPERIMENTAL STUDIES WITH SPECIES OF BAUHINIA (FABACEAE) TO EVALUATE THE ANTIDIABETIC ACTIVITY
}

\author{
PAULA ${ }^{1}$, C. S.; MIGUEL ${ }^{1}$, M. D. \\ 1 - Departamento de Farmácia. Universidade Federal do Paraná - UFPR. Curitiba, PR, Brasil \\ Autor para correspondência: crisspaula@onda.com.br
}

\section{RESUMO:}

Modelos de estudo in vivo e in vitro e em enzimas/receptores são utilizados quando se pretende comprovar a ação farmacológica de um determinado extrato e/ou princípio ativo obtido de plantas, além de elucidar o mecanismo de ação. O objetivo deste estudo foi realizar um levantamento da literatura sobre quais os ensaios que já foram realizados com plantas do gênero Bauhinia L. para verificar a atividade hipoglicemiante, e quais as espécies pesquisadas. Para isso, foi realizado uma revisão da literatura através de busca eletrônica nas bases de dados Pubmed, Science direct, Lilacs e na biblioteca eletrônica Scielo, publicados no período de 1995 à 2015 (20 anos), utilizando como descritores "Bauhinia" and "extract" and "hypoglycemic" or "antidiabetic" seus correspondentes em português, "Bauhinia" e "extrato" e "hipoglicêmica" ou "antidiabético". Foram localizados artigos relacionados à pesquisa com doze espécies de Bauhinia L. A Bauhinia forficata é a que mais apresenta estudos sobre o tema, sendo localizado 13 artigos, seguida da B. variegata com 4 estudos. Foi possível verificar que pouco se conhece sobre a atividade hipoglicemiantes das espécies de Bauhinia L., e que mais estudos são necessários para comprovar o uso popular.

Palavras-chave: pata de vaca, planta medicinal, diabetes

\begin{abstract}
:
In vivo and in vitro and in enzymes / receptors study models are used when you want to prove the pharmacological action of a particular extract and / or active ingredient from plants, as well as elucidate the mechanism of action. The aim of this study was to survey the literature on which trials have already been conducted with the genus Bauhinia L. plans to check the hypoglycemic activity, and what the surveyed species. For this, we conducted a literature review through electronic search on Pubmed, Science direct, Lilacs and electronic library SciELO, published from 1995 to 2015 (20 years), using as keywords "Bauhinia" and "extract "and" hypoglycemic "or" Antidiabetic "their equivalents in Portuguese," Bauhinia "and" extrato "and" hipoglicêmico "or" anti-diabético". Research-related articles were found with twelve species of Bauhinia L. The Bauhinia forficata is the one that presents studies on the subject, being located 13 articles, followed by $B$. variegata , 4 studies. It observed that little is known about the hypoglycemic activity of the species of Bauhinia L., and that further studies are needed to confirm the popular use.
\end{abstract}

Keywords: cow's paw, medicinal plant, diabetes 


\section{INTRODUÇÃO}

Modelos de estudo in vivo e in vitro e em enzimas/receptores são utilizados quando se pretende comprovar a ação farmacológica (GUIDO, ANDRICOPULO, OLIVA, 2010) de um determinado extrato e/ou princípio ativo obtido de plantas, além de elucidar o mecanismo de ação. Os estudos in vitro são geralmente mais rápidos, menos onerosos, utilizam pequena quantidade de material, as variáveis experimentais podem ser mais facilmente controladas e fornecem resultados quantitativos. Já os testes in vivo podem confirmar o uso popular de determinada espécie, fornecer informações preliminares a respeito de efeitos tóxicos e permitir a análise de fatores que interferem na ação dos princípios ativos da planta, como por exemplo, a influência das vias de administração (CALIXTO, 2001). Neste contexto, as plantas medicinais sempre foram objeto de estudo na tentativa de descobrir novas fontes de obtenção de princípios ativos para o desenvolvimento de medicamentos.

Plantas do gênero Bauhinia L. são exemplos de espécies que vem sendo estudadas na tentativa de se identificar compostos com potencial terapêutico. Estas plantas são pertencentes à família Fabaceae, encontradas nas áreas tropicais do planeta (CECHINEL FILHO, 2009). São classificadas em 350 espécies (THE PLANT LIST, 2013) sendo 59 com registros de serem encontradas no Brasil, com domínios fitogeográficos principalmente na Amazônia, Caatinga, Cerrado, Mata Atlântica, Pampa e Pantanal (VAZ, 2014). São conhecidas como "pata-de-vaca" (CORRÊA, 1952) tendo em vista o formato de suas folhas que lembram o rastro de bovinos.

Algumas espécies podem ser utilizadas na recuperação de áreas degradadas (LORENZI, 1991), na arborização pública como ornamentais, e por suas propriedades medicinais, utilizada como hipoglicemiante, hipocolesterolêmica, expectorante, depurativa, diurética, antidiarreica, anti-infecciosa e em processos dolorosos (SILVA; CECHINEL-FILHO, 2002). O uso popular medicinal mais comum descrito pela literatura é na redução da glicemia observada em várias espécies de Bauhinia L., sendo geralmente utilizadas por pessoas diabéticas na forma de chá (LORENZI; MATOS, 2008).

Considerando a importância de estudos que reafirmem os benefícios e comprovações de eficácia das plantas medicinais além da possibilidade de servirem de fonte para o desenvolvimento de novos medicamentos, o objetivo deste estudo foi realizar um levantamento da literatura sobre quais os ensaios in vitro e in vivo e em enzimas/receptores que já foram realizados com plantas do gênero Bauhinia L. para verificar a atividade hipoglicemiante, e quais as espécies pesquisadas.

\section{METODOLOGIA}

Trata-se de uma revisão da literatura sobre quais foram os ensaios in vivo e in vitro e em enzimas/receptores realizados com plantas do gênero Bauhinia L., e quais as espécies pesquisadas, que avaliaram a atividade hipoglicemiante, através de busca 
eletrônica nas bases de dados Pubmed, Science direct, Lilacs e na biblioteca eletrônica Scielo. A busca limitou-se a artigos escritos em inglês e português publicados no período de 1995 a 2015 (20 anos), utilizando como descritores "Bauhinia" and "extract" and "hypoglycemic" or "antidiabetic" seus correspondentes em português, "Bauhinia" e "extrato" e "hipoglicêmica" ou "antidiabético". Foram incluídos, após leitura de títulos e resumos, os artigos que se encaixavam no tema e como critérios de exclusão, adotaram-se artigos que não apresentassem nenhum ensaio laboratorial in vitro e in vivo e em enzimas/receptores com espécies do gênero Bauhinia ou que estivessem redigidos em outras línguas que não as anteriormente citadas ou que se apresentassem em duplicata na mesma base. No caso do título e resumo não serem suficientes para a inclusão ou exclusão, o artigo foi lido na íntegra.

\section{RESULTADOS E DISCUSSÃO}

A pesquisa realizada, utilizando os critérios acima descritos, encontrou 14 artigos na base Pubmed, 106 na Science direct, 5 na Lilacs e 3 na Scielo. Deste total de artigos localizados em cada base, por não estarem relacionados ao assunto pesquisado, ensaios in vivo e in vitro e em enzimas/receptores realizados com plantas, foram excluídos 2 artigos da Pubmed, 91 da Science Direct, 3 da Lilacs e 1 na Scielo. $O$ restante, 31 artigos foram utilizados na pesquisa, e apresentados na Tabela 1, em que estão listadas as espécies de Bauhinia L. que possuem ensaios relacionados à atividade hipoglicemiante e respectivas fontes de referência.

TABELA 1- ENSAIOS in vivo $\mathrm{E}$ in vitro REALIZADOS COM PLANTAS DO GÊNERO Bauhinia L.

\begin{tabular}{|c|c|c|}
\hline $\begin{array}{l}\text { ATIVIDADE } \\
\text { AVALIADA }\end{array}$ & ESPÉCIE & REFERÊNCIAS \\
\hline Hipoglicemiante & $\begin{array}{l}\text { B. Kalbreyeri } \\
\text { B. megalandra } \\
\text { B. monandra } \\
\text { B. purpurea } \\
\text { B. thonningii } \\
\text { B. tomentosa } \\
\text { B. vahlii } \\
\text { B. variegata }\end{array}$ & $\begin{array}{r}\text { LEMUS et al., 1999; FUENTES, ARANCIBIA-AVILA, } \\
\text { ALARCÓN, 2004; FUENTES, ALARCÓN , } 2006 \\
\text { ALMEIDA et al., } 2006 \\
\text { ROMÁN-RAMOS et al., } 1992 \\
\text { RUSSO et al., 1990; SILVA et al.., 2002a; PEPATO et } \\
\text { al., 2002, JORGE et al., 2004; SOUZA et al., 2004; } \\
\text { LINO et al., 2004; VASCONCELOS et al., 2004; } \\
\text { MENEZES et al., 2007; CUNHA et al., 2010; CURCIO } \\
\text { et al., 2012; CAZAROLLI et al., 2013; FARAG et al., } \\
\text { 2015. } \\
\text { MURILO et al., 2006 } \\
\text { GONZALEZ-MUJICA et al., 2003; ESTRADA et al., } \\
\text { 2005; FERNÁNDEZ-PENA et al., 2008 } \\
\text { MENEZES et al., 2007 } \\
\text { MARTINEZ et al., 2009; MESHRAM et al.,, 2013 } \\
\text { OJEZELE, ABATAN, } 2011 \\
\text { DEVAKI et al.,, 2011 } \\
\text { NARAYAN et al., } 2012 \\
\text { FRANKISH et al.,, 2010; VADIVEL et al., 2011; } \\
\text { KUMAR et al., 2012; GAUTAM et al., } 2012\end{array}$ \\
\hline
\end{tabular}

FONTE:AAutora (2015)

Visão Acadêmica, Curitiba, v.16, n.3, Jul. - Set./2015 - ISSN 1518-8361 
Foram localizados artigos relacionados à pesquisa com doze espécies (Tabela 1) de Bauhinia L., que corresponde a $3,43 \%$ das espécies catalogadas. A Bauhinia forficata é a que mais apresenta estudos sobre o tema, sendo localizado 13 artigos, seguida da $B$. variegata com 4 estudos.

Nos experimentos que verificaram a atividade hipoglicemiante dos extratos in vivo, foram utilizadas para indução do diabetes nos animais duas substâncias muito conhecidas, a estreptozotocina (EZT) e o aloxano (ALX). A EZT é um quimioterápico, utilizado no tratamento de tumores do pâncreas, que reduz a ação da adeninanicotinamida das células $\beta$-pancreáticas, inibindo a síntese de DNA, consequentemente, bloqueando de forma irreversível a produção de insulina. Causa grave hiperglicemia, induzindo o diabetes melitus nos animais, semelhante ao diabetes tipo 1 em humanos (CARVALHO et al., 2003). O ALX destroi células $\beta$ - pancreáticas (SILVA et al., 2002). Para os experimentos listados foram utilizados principalmente ratos com diabetes induzido por EZT (PEPATO) et al., 2002; CUNHA et al., 2010; NARAYAN et al., 2012 ) e ALX em coelhos e ratos (LEMUS et al., 1999; SILVA et al., 2002; JORGE et al., 2004; FUENTES, ARANCIBIA-AVILA, ALARCÓN, 2004; SOUZA et al., 2004. LINO et al., 2004; ALMEIDA et al., 2006; DEVAKI et al., 2011). Além disso, pacientes diabéticos também testaram um extrato do gênero (RUSSO) et al., 1990).

De acordo com os autores um dos mecanismos de ação investigados foi a redução da absorção de glicose intestinal em ratos (GONZALEZ-MUJICA et al., 2003) com inibição da enzima a-Glucosidase (FARAG et al., 2015). As a -glucosidades são respresentadas pelas enzimas intestinais $\alpha$-amilase, trealose-6-fosfato hidrolase, sacarase e maltase. Elas retardam a digestão de carboidratos e, consequentemente, promovem melhor controle nas concentrações de glucose após as refeições. Inibidores de glucosidases são agentes de grande interesse terapêutico, e fazem parte do arsenal de medicamentos utilizados para o tratamento do diabetes tipo II, como por exemplo, a acarbose (MELO, CARVALHO, 2006).

Também atuam por inibição da glicose-6-fosfatase (G-6-Pase) (ESTRADA et al., 2005) que é um sistema enzimático do retículo endoplasmático responsável pela hidrólise da glicose-6-fosfato e a consequente formação de glicose nos órgãos que a possuem (fígado, rim e intestino) (SANTOS-ANTUNES, FONTES, 2009). Quando a enzima é inibida, observa-se redução da glicose.

Alguns extratos foram testados para se verificar um possível efeito insulinomimético, ou seja, semelhante ao da insulina, que regula a homeostase de glicose em vários níveis, reduzindo a produção hepática (via redução da gliconeogênese e glicogenólise) e aumentando a captação periférica, principalmente nos tecidos muscular e adiposo (CARVALHEIRA et al., 2002; JORGE et al., 2004) além disso, o efeito de estimulação da recaptação de glicose em glândulas gástricas isoladas 
isoladas de coelhos também foi realizado (FUENTES, ALARCÓN, 2006). Em alguns experimentos que utilizaram camundongos normoglicêmicos, também foi observando hipoglicemia (MENEZES et al., 2007) ou como sugerido por Silva et al.(2002) aumento do consumo de glicose periférica.

\section{CONCLUSÃO}

A partir dos resultados encontrados, é possível verificar que pouco se conhece sobre a atividade hipoglicemiante das espécies de Bauhinia L., e que mais estudos são necessários para comprovar o uso popular. Os ensaios preliminares são utilizados para direcionar a realização de ensaios clínicos e consistem de dados relacionados principalmente à eficácia, demonstrada através de experimentos em enzimas/receptores, in vitro e em modelos animais, sendo fundamentais para a consolidação do uso de determinada espécie.

\section{REFERÊNCIAS}

ALMEIDA, E. R.; GUEDES, M. C.; ALBUQUERQUE, J. F.; XAVIER, H. Hypoglycemic effect of Bauhinia cheilandra in rats. Fitoterapia, v. 77, p. 276-278, 2006.

CALIXTO, J. B. Estudos farmacológico pré-clínicos de plantas medicinais. In: YUNES, R. A.; CALIXTO, J. B. Plantas Medicinais sob a ótica da Química Medicinal Moderna. Chapecó: Ed. Argos, 2001. p. 82-84.

CARVALHEIRA, J. B. C.; ZECCHIN, H. G.; SAAD, M. J. A. Vias de Sinalização da Insulina. Arq Bras Endocrinol Metab. v. 46, n. 4, p. 419-425. 2002.

CARVALHO, E. N.; CARVALHO, N. A. S.; FERREIRA, L. M. Experimental model of induction of diabetes mellitus in rats. Acta Cir. Bras. v. 18, p. 60-64. 2003.

CAZAROLLI, L. H.; PEREIRA, D. F.; KAPPEL, V. D.; FOLADOR, P.; FIGUEIREDO, M. S. R. B.; PIZZOLATTI, M. G.; SILVA, F. R. M. B. Insulin signaling: A potential signaling pathway for the stimulatory effect of kaempferitrin on glucose uptake in skeletal muscle. Eur J Pharmacol. v. 712, p. 1-7, 2013.

CECHINEL FILHO, V. Chemical Composition and Biological Potential of Plants from the Genus Bauhinia. Phytother Res. v. 23, p. 1347-1354, 2009

CORREAA, M.P. Dicionário das plantas úteis do Brasil e das exóticas cultivadas. Rio de Janeiro: Imprensa Nacional, v. 3, 1952. 
CURCIO, S. A.; STEFAN, L. F.; RANDI, B. A.; DIAS, M. A.; DA SILVA, R. E.; CALDEIRA, E. J. Hypoglycemic effects of an aqueous extract of Bauhinia forficata on the salivary glands of diabetic mice. Pak J Pharm Sci. v. 25, n. 3, p. 493-9, 2012.

CUNHA, A. M.; MENON, S.; MENON, R.; COUTO, A. G.; BURGER, C.; BIAVATTI, M. W. Hypoglycemic activity of dried extracts of Bauhinia forficata Link. Phytomedicine, v. 17, p. $37-41,2010$.

DEVAKI, K.; BEULAH, U.; AKILA, G.; NARMADHA, R.; GOPALAKRISHNAN, V. K. Glucose lowering effect of aqueous extract of Bauhinia tomentosa $L$. on alloxan induced type 2 diabetes mellitus in wistar albino rats. J Basic Clin Pharm. v. 2, n. 4, p. 167-174, 2011.

ESTRADA, O.; HASEGAWA, M.; GONZALEZ-MUJÍCA, F.; MOTTA, N.; PERDOMO, E.; SOLORZANO, A.; MÉNDEZ, J.; MÉNDEZ, B.; ZEA, E. G. Evaluation of flavonoids from Bauhinia megalandra leaves as inhibitors of glucose-6-phosphatase system. Phytother Res. v. 19, n. 10, p. 859-63, 2005

FARAG, M. A.; SAKNA, S. T.; EL-FIKY, N. M.; SHABANA, M. M.; WESSJOHANN, L. A. Phytochemical, antioxidant and antidiabetic evaluation of eight Bauhinia L. species from Egypt using UHPLC-PDA-qTOF-MS and chemometrics. Phytochemistry. v. 119, p. $41-50,2015$.

FERNÁNDEZ-PEÑA, C.; GONZÁLEZ-MUJICA, F.; MOTTA, N. ; TILLETT, S. Effect of aqueous extract of leaves of Bauhinia megalandra glucogenólisis on the liver in rats. Arch. venez. farmacol. ter, v. 27, n. 2, p. 129-131, 2008.

FRANKISH, N.; MENEZES, F. S.; MILLS, C.; SHERIDAN, H. Enhancement of insulin release from the beta-cell line INS-1 by an ethanolic extract of Bauhinia variegata and its major constituent roseoside. Planta Med. v. 76, n. 10, p. 995-997, 2010.

FUENTES, O.; ARANCIBIA-AVILA, P.; ALARCÓN, J. Hypoglycemic activity of Bauhinia candicans in diabetic induced rabbits. Fitoterapia. v. 75, p. 527-532, 2004.

FUENTES, O.; ALARCÓN, J. Bauhinia candicans stimulation of glucose uptake in isolated gastric glands of normal and diabetic rabbits. Fitoterapia. v. 77, p. 271-275, 2006.

GAUTAM, B.; VADIVEL, V.; STUETZ, W.; BIESALSKI, H. K. Bioactive compounds extracted from Indian wild legume seeds: antioxidant and type II diabetes-related enzyme inhibition properties. Int J Food Sci Nutr.. v. 63, n. 2, p. 242-245, 2012. 
GONZALEZ-MUJICA, F.; MOTTA, N.; MÁRQUEZ, A. H.; CAPOTE-ZULUETA, J. Effects of Bauhinia megalandra aqueous leaf extract on intestinal glucose absorption and uptake by enterocyte brush border membrane vesicles. Fitoterapia. v. 74, n. 1-2, p. 84-90, 2003.

GUIDO, R. V. C.; ANDRICOPULO, A. D.; OLIVA, G. Planejamento de fármacos, biotecnologia e química medicinal: aplicações em doenças infecciosas. Estudos Avançados, v. 24, n. 70, p. 81-98, 2010.

JORGE, A. P.; HORST, H.; SOUSA, E.; PIZZOLATTI, M. B.; SILVA, F. R. M. B. Insulinomimetic effects of kaempferitrin on glycaemia and on 14C-glucose uptake in rat soleus muscle. Chem Biol Interact.. v. 149, p. 89-96, 2004.

KUMAR, P.; BARAIYA, S.; GAIDHANI, S. N.; GUPTA, M. D.; WANJARI, M. M. Antidiabetic activity of stem bark of Bauhinia variegata in alloxan-induced hyperglycemic rats. J Pharmacol Pharmacother. v. 3, n. 1, p. 64-66, 2012.

LEMUS, I.; GARCÍA, R.; DELVILLAR. E.; KNOP, G. Hypoglycaemic activity of four plants used in Chilean popular medicine. Phytother Res.. v. 13, p. 91-94, 1999.

LINO, C. S.; DIÓGENES, J. P. L.; PEREIRA, B. A.; FARIA, R. A. P. G.; ANDRADE NETO, M. ALVES, R. S.; QUEIROZ, M. G. .R.; SOUSA, F.C.S.; VIANA, G. S. B. Antidiabetic Activity of Bauhinia forficata Extracts in Alloxan-Diabetic Rats. Biol. Pharm. Bul. v. 27, n. 1, p. 125-127, 2004.

LORENZI, H. Árvores brasileiras. Manual de identificação. Editora Plantarum Ltda. Nova Odessa, São Paulo, 1991, 352p.

LORENZI, H.; MATOS, F. J. A. Plantas Medicinais no Brasil: Nativas e Exóticas. 2a. ed. Nova Odessa, SP: Instituto Plantarum, 2008, 544p.

MARTINEZ, N.; CAVAMA, E.; GONÇALVEZ, L.; LABRADOR, S.; ESPINO, C.; PÉREZ, L. Hipoglicemic effect os Bauhinia purpúrea L. in euglicemic mice. Comunidad y Salud, v. 7, n. 2, p. 45-51, 2009.

MELO, E. B.; CARVALHO, I. a e $\beta$-glucosidases como alvos moleculares para desenvolvimento de fármacos. Quím. Nova. v. 29, n.4, p. 840-843, 2006.

MENEZES, F. S.; MINTO, A. B. M.; RUELA, H. S.; KUSTER, R. M.; SHERIDAN, H.; FRANKISH, N. Hypoglycemic activity of two Brazilian Bauhinia species: Bauhinia forficata L. and Bauhinia monandra Kurz. Brazilian Journal of Pharmacognosy. v. 17, n. 1, p. 08-13, 2007. 
MENEZES, F. S.; MINTO, A. B. M.; RUELA, H. S.; KUSTER, R. M.; SHERIDAN, H.; NEIL, F. Hypoglycemic activity of two Brazilian Bauhinia species: Bauhinia forficata L. and Bauhinia monandra Kurz. Rev. bras. farmacogn. v. 17, n. 1, p. 8-13, 2007.

MESHRAM, S. S. ; ITANKAR, P. R.; PATIL, A. T. To Study Antidiabetic Activity of Stem Bark of Bauhinia purpurea Linn. J Pharmacogn Phytochem. v. 2, n. 1, p. 171-175, 2013.

MURILLO, E.; TIQUE, M. M.; OSPINA, L. F.; LOMBO, O. Preliminary evaluation of hypoglycemic activity in diabetic mice and in vitro antioxidant activity of Bauhinia kalbreyeriHarms extracts. Rev. colomb. cienc. quim. farm. v. 35, n. 1, p. 64-80; 2006.

NARAYAN, D. S.; JAGANNATH, P. V.; CHANDRA, D. S. Evaluation of AntiInflammatory, Anti-diabetic activityof Indian Bauhinia vahlii (stembark). Asian Pac J Trop Biomed.. p. 1382-1387, 2012.

OJEZELE, M. O.; ABATAN, O. M. Hypoglycaemic and coronary risk index lowering effects of Bauhinia thoningii in alloxan induced diabetic rats. African Health Sciences, v. 11, n. 1, p. 85-89, 2011.

PEPATO, M. T.; KELLER, E. H.; BAVIERA, A. M.; KETTELHUT, I. C.; VENDRAMINI, R. C.; BRUNETTI, I. L. Anti-diabetic activity of Bauhinia forficata decoction in streptozotocin-diabetic rats. J Ethnopharmacol. v. 81, n. 2, p. 191-197, 2002.

ROMÁN-RAMOS, R.; ALARCÓN-AGUILAR, F.; LARA-LEMUS, A.; FLORES-SAENZ, J. L. Hypoglycemic effect of plants used in Mexico as antidiabetics. Arch Med Res. v. 23, p. 59-64, 1992.

RUSSO, E. M.; REICHELT, A. A.; DE-SÁ, J. R.; FURLANETTO, R. P.; MOISÉS, R. C.; KASAMATSU, T. S.; CHACRA, A. R. Clinical trial of Myrcia uniflora and Bauhinia forficata leaf extracts in normal and diabetic patients. Braz J Med Biol Res. v. 23, n. 1, p. $11-20,1990$

SANTOS-ANTUNES, J.; FONTES, R. Glicogenose Tipo I. Disfunção do Complexo Glicose-6-fosfátase. Arq Med. v. 23, n. 3, p.109-117, 2009.

SILVAF. R. M. B.; SZPOGANICZB.; PIZZOLATTI M. G.; WILLRICH M. A. V.; SOUSAE. Acute effect of Bauhinia forficata on serum glucose levels in normal and alloxan-induced diabetic rats. J. Ethnopharmacol. v. 83, p. 33-37, 2002a. 
SILVA, K. L., CECHINEL-FILHO, V. Plantas do Gênero Bauhinia: Composição Química e Potencial Farmacológico. Quím Nova. v.25, n. 3, p.449-454, 2002.

SOUSA, E.; ZANATTA, L.; SEIFRIZ, I.; CRECZYNSKI-PASA, T. B.; PIZZOLATTI, M. G.; SZPOGANICZ, B.; SILVA, F. R. M. B. Hypoglycemic effect and antioxidant potential of kaempferol-3,7-O-( $\alpha$ )-dirhammnoside from Bauhinia forficata leaves. J Nat Prod. v. 67, p. 829-832, 2004.

THE PLANT LIST (2013). Version 1.1. Disponível em:<http://www.theplantlist.org> Acesso em: 01/01/2014

VADIVEL, V.; STUETZ, W.; SCHERBAUM, V.; BIESALSKI, H.K. Total free phenolic content and health relevant functionality of Indian wild legume grains: Effect of indigenous processing Methods. J Food Compost Anal.. v. 24, p. 935-943, 2011.

VASCONCELOS, F.; SAMPAIO, S. V.; GARÓFALO, M. A.; GUIMARÃES, L. F.; GIGLIO, J. R.; ARANTES, E. C. Insulin-like effects of Bauhinia forficata aqueous extract upon Tityus serrulatus scorpion envenoming. J Ethnopharmacol. v. 95, n. 2-3, p. 385-92, 2004.

VAZ, A. M. S. F. Bauhinia in Lista de Espécies da Flora do Brasil 2014. Jardim Botânico do Rio de Janeiro. Disponível em: http://floradobrasil.jbrj.gov.br/jabot/floradobrasil/ FB22831. Acesso em 05/03/2014. 